\title{
Maropitant Citrate
}

National Cancer Institute

\section{Source}

National Cancer Institute. Maropitant Citrate. NCI Thesaurus. Code C78028.

A neurokinin receptor antagonist with antiemetic activity. This agent is approved for veterinary use only. 